\title{
An Adaptive Deformable Template for Mouth Boundary Modeling
}

\author{
Ali Reza Mirhosseini ${ }^{1}$, Kin-Man Lam ${ }^{2}$, and Hong $\operatorname{Yan}^{1}$ \\ 1 Department of Electrical Engineering, University of Sydney, Australia \\ 2 Electrical Engineering Department, Hong Kong Polytechnic University, Hong Kong
}

\begin{abstract}
The authors propose an algorithm to automatically extract mouth boundary model in human face images using deformable templates. Our vision algorithm is based on a hierarchical model adaptation scheme. In this paper it will be shown that the role of priori knowledge of the domain is essential for perceptual organization in our algorithm. The knowledge about the shape of the object is used to define its initial deformable template. Each mouth boundary curve is initially formed based on three control points whose locations are found through an optimization process using a suitable cost functional. The cost functional captures the essential knowledge about the shape to perceptually organize image information. Two of the control points are the mouth cormers that are primarily located using the priori knowledge of the properties of edge map of the mouth image at its corners. They are used as the initial location of the mouth after an approximate mouth window is found based on locating the head boundary. The model is hierarchically improved in the second stage of the algorithm. Each boundary curve is finely tuned using more control points. An old model is adaptively replaced by a new model only if a secondary cost is further reduced. The results show that model adaptation technique satisfactorily enhances the mouth boundary model in an automated fashion.
\end{abstract}

\section{Introduction}

Modeling natural and flexible objects is a complicated task that has not been thoroughly investigated. For instance automatic detection and description of salient features is crucially important in building a human face image processing and analysis system. This difficult task was initiated by [3] and continued by many others $[2,1,7]$. Conventional edge detectors are not able to find the boundary of the natural objects especially facial features such as mouth and eyes partially because the edges are seldom sharp as the idealized detectable edges by edge detectors. Furthermore the contrast of gray level around the boundaries is very low. However even the local edges exist they still can not be organized into a global percept [7]. Recent works by [1] using active contours and deformable models pioneered modeling the shape of boundary for natural objects. Deformable models make use of global information, and hence increase the reliability of locating a contour. A deformable model is specified by a set of parameters, which allows inclusion of a priori knowledge of the expected shape of an object. 


\section{Mouth Deformable Model}

A mouth can have large degrees of variations due to its physical flexibility. Existence of various facial expressions together with its shape variations among individuals make modeling the shape of mouth contours difficult by rigid templates. Therefore it seems appropriate to deal with these problems using deformable templates [7]. A mouth model can be represented by three boundary curves passing over inner and outer mouth outlines. Each curve passes through a number of control points that can be found through optimizing a suitably defined cost function that includes the required knowledge and constraints.

\subsection{Adaptive Modeling Procedure}

An automated procedure to produce a mouth model can be described as:

1. Locate approximate mouth corners as the initial location of the mouth,

2. Initialize mouth model using three parametric curves with three control points for each boundary curve,

3. Optimize the model for the best control points using a primary cost function. The search for a better control point takes place within a neighborhood window. Replace the old model with new model if cost further decreases,

4. Add more control points and go to step 3 if the model was renewed earlier, otherwise end the procedure.

The order of the mouth outline curves and the position of their control points are the major parameters that are to be optimized. We propose an order adaptation algorithm for fine tuning the model using higher order models for mouth outline curves. Finding the model parameters is cast into an optimization problem.

\section{Mouth Corner Detection}

The approximate location of a mouth can be worked out with anthropometric standards, using extracted head boundaries $[6,10]$. The mouth boundaries are located between two mouth corners, denoted by $\mathbf{X}_{\mathbf{0}}$ and $\mathbf{X}_{\mathbf{1}}$ as illustrated in Fig.2. These two points can form the initial location of the mouth. A corner detection method is used based on the scheme proposed by [4] and applied by [9] to facial corners. It provides information about corner orientations and location. A corner is formed by intersection of two straight lines. A detected corner points is classified as a mouth corner candidate if it satisfies certain properties that are based on image information, as tabulated in Table.1.The curvature $\beta$ is defined as the acute angle between the two lines. The orientation $\alpha$ represents the orientation of the line which bisects the acute angle between the two lines. The region dissimilarity, $D$, is a measure of the difference between the gray level averages in the two regions $R_{1}$ and $R_{2}$ bounded by the edges [9]. The first step for corner detection is to detect the edges of an image. Each edge pixel is then considered as a candidate for a corner. In order to detect the two lines of a 


\begin{tabular}{|c|c|c|c|}
\hline & $\beta$ & $\alpha$ & $D$ \\
\hline$C_{0}$ & $52.5^{\circ}-90^{\circ}$ & $-45^{\circ}-45^{\circ}$ & $<-10$ \\
\hline$C_{1}$ & $52.5^{\circ}-90^{\circ}$ & $135^{\circ}-225^{\circ}$ & $<-10$ \\
\hline
\end{tabular}

Table 1. Properties of the corners of the mouth.

corner accurately we use the matching scheme used in [9]. This method has the advantage of being insensitive to noisy edge pixels. The existence of noisy edge pixels will not affect the identification of the lines and the corner features.

\subsection{Selection and Detection of Corner Positions}

A procedure is introduced to simplify the selection of the best corner among corner candidates [9]. The candidates for a corner type are clustered into groups. Two points, $\left(x_{i}, y_{i}\right)$ and $\left(x_{j}, y_{j}\right)$, of the same corner type belong to the same cluster if

$$
\left|y_{i}-y_{j}\right|+\left|x_{i}-x_{j}\right|<d
$$

where $d$ is a threshold. The representative of a cluster is the one with the largest $|D|$. The next procedure is to choose one of the clusters to represent the corner. In this procedure, different cost functions are defined for the different corner types.

By assuming that the face is rotated to a limited degree, $d x=x_{1}-x_{0}$ represents the approximate length of the mouth. Pairs of corners are thus formed if

$$
0.3 \times \text { face_width }<d x<0.5 \times \text { face_width }
$$

where face_width is the width of the face which is obtained from the face boundary. A pair is then selected to represent the two corners if the value of the following cost function is a minimum.

$$
\cos t=K_{1} \frac{|d y|}{|d y|_{\max }}+K_{2} \frac{|D|_{\max }}{|D|}
$$

where $K_{1}$ and $K_{2}$ are the weighting factors for the two normalized cost terms, $|d y|_{\text {max }}$ is the maximum value of $|d y|=\left|y_{1}-y_{0}\right|$; and $|D|_{\text {max }}$ is the maximum value of $|D|=\left|D\left(C_{0}\right)+D\left(C_{1}\right)\right|$ among all the corner pairs.

\section{Initial Mouth Model}

The initial mouth model is composed of three mouth outline curves, denoted by $P_{1}, P_{2}$ and $P_{3}$ and illustrated in Fig.2. Each curve is initially a parabola with three control points including $\mathbf{X}_{0}$ and $\mathbf{X}_{1}$ and a third point, $\mathbf{X}_{2}$, which is to be located through optimizing a cost function. 


\subsection{Cost function for Mouth Shape}

An optimization process is employed to precisely locate mouth corners and find mouth boundaries for the initial mouth model. We use a primary cost function based on a set of potential energy functions, composed of a set of conditions that suitably constraints each mouth outline curve. Each potential energy captures the features of each mouth boundary and its relation with the other parts of the mouth model. These features include distance between mouth outlines, corner point potential, and edge and valley potentials.

The total primary potential energy $E 1_{P_{2}}$ for each boundary curve $P_{i}$ formed by three control points $\mathbf{X}_{\mathbf{i}}, \mathbf{X}_{\mathbf{j}}$ and $\mathbf{X}_{\mathbf{k}}$ is defined as:

$$
E 1_{P_{\imath}}\left(\mathbf{X}_{\mathbf{i}}, \mathbf{X}_{\mathbf{j}}, \mathbf{X}_{\mathbf{k}}\right)=E_{c}\left(P_{i}, P_{j}\right)+E_{d 1}+E_{e}\left(P_{i}, \mathbf{X}_{\mathbf{i}}, \mathbf{X}_{\mathbf{j}}\right)+E_{v}\left(P_{i}, \mathbf{X}_{\mathbf{i}}, \mathbf{X}_{\mathbf{j}}\right)
$$

where

1. The corner potential term, $E_{c}$, is a measure of the fitness of a point to be a mouth corner. The potential is defined as being equal to the region dissimilarity of the corner at the point:

$$
E_{c}\left(P_{i}, P_{j}\right)=-\left|D\left(R_{1}, R_{2}\right)\right|
$$

where $R_{1}$ and $R_{2}$ are the two regions in the window separated by the two boundary curves $P_{i}$ and $P_{j}$.

2. The valley energy term, $E_{v}$, is given as the integral of valley forces $\Phi_{v}$ along boundary curve $P_{i}$ between two mouth corners $\mathbf{X}_{\mathbf{i}}$ and $\mathbf{X}_{\mathbf{j}}$ :

$$
E_{v}\left(P_{i}, \mathbf{X}_{\mathbf{i}}, \mathbf{X}_{\mathbf{j}}\right)=-\frac{1}{L_{P_{i}}} \int_{P_{i}} \Phi_{e}(\mathbf{x}) d s
$$

3. The edge potential term, $E_{e}$, is a measure of the edge intensities $\Phi_{e}$ along boundary curve $P_{i}$ between two mouth corners $\mathbf{X}_{\mathbf{i}}$ and $\mathbf{X}_{\mathbf{j}}$ :

$$
E_{e}\left(P_{i}, \mathbf{X}_{\mathbf{j}}, \mathbf{X}_{\mathbf{j}}\right)=-\frac{1}{L_{P_{\mathbf{v}}}} \int_{P_{\mathbf{v}}} \Phi_{e}(\mathbf{x}) d s
$$

4. Distance potential term, $E_{d 1}$, is defined in order to control the shape of the mouth models:

$$
E_{d 1}=\left|h_{t b}-d\left(\mathbf{x}_{i}, \mathbf{x}_{j}\right)\right|+K_{3}\left|b-b_{1}\right|+K_{4}\left|b-b_{2}\right|
$$

where $h_{t b}=\lambda\left|\mathbf{X}_{\mathbf{1}}-\mathbf{X}_{\mathbf{0}}\right|$. The definitions of these two potentials are illustrated in Fig.1 (a). It measures the absolute difference between the expected thickness $h_{t b}$ of the upper or lower lip and the corresponding thickness $d\left(P_{i}, P_{j}\right)$ as represented by the model. $K_{3}$ and $K_{4}$ are the weighting factors for their corresponding terms. 


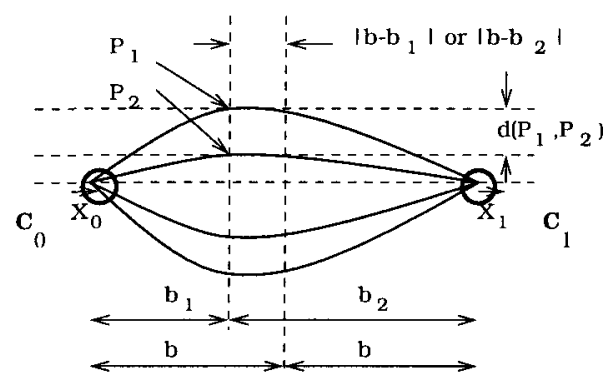

(a)

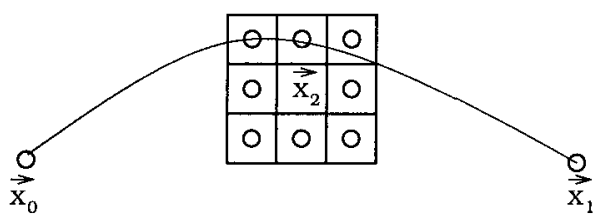

(b)

Fig. 1. (a) Dimensions of the mouth for measuring $E_{1}$ and $E_{2}$ and two mouth corners are $C_{0}$ and $C_{1}$, (b) Neighbors searched in the greedy algorithm.

where $L_{P_{2}}$ denotes the length of the parametric curve $P_{i}$ in pixels and the edge intensities and valley forces, $\Phi_{e}$ and $\Phi_{v}$, are extracted by morphological edge and valley detectors [5].

Distance potential, $E_{d}\left(P_{i}, P_{j}\right)$, is a measure of the shape of the mouth and controlling the distance between two mouth boundaries and is defined with the minimum distance between $P_{i}$ and $P_{j}$.

\subsection{Search for Cost Minimization}

The minimum value of $E_{P_{2}}$ is found by moving the control points of each outline curve. The total potential energy for each mouth outline curve is optimized by searching for the control points within a neighborhood region around each curve. The search process is based on a greedy algorithm that is applied in active contour model optimization technique as shown in Fig.1 (b) [10]. Each control point can move to an eight connected neighborhood window if the total potential energy decreases. After the initial model is optimized in this step, it is passed to step 4 of procedure in Section 2.1., for fine tuning.

\section{Adapting Mouth Model}

The old model extracted in step 3 of procedure in Section 2.1., can be improved by increasing the order of each boundary curve, through adding more control points to each mouth outline curve. Therefore for instance a cubic spline curve, as a new model, can be formed using four control points for each outline curve, by adding an extra control point to an old parabolic boundary curve model.

The total secondary potential energy $E 2_{P_{z}}$ for a generalized new model with a boundary curve $P_{i}$ formed using $m$ control points $\left\{\mathbf{X}_{\mathbf{1}}, \cdots, \mathbf{X}_{\mathbf{m}-1}\right\}$ is defined as:

$E 2_{P_{\imath}}\left(\mathbf{X}_{\mathbf{1}}, \cdots, \mathbf{X}_{\mathbf{m}-1}\right)=E_{e}\left(P_{i}, \mathbf{X}_{\mathbf{1}}, \cdots, \mathbf{X}_{\mathbf{m}-1}\right)+E_{v}\left(P_{i}, \mathbf{X}_{\mathbf{1}}, \cdots, \mathbf{X}_{\mathbf{m}-\mathbf{1}}\right)+E_{d 2}$ where 
1. The valley energy term, $E_{v}$, is given by Equation (7),

2. The edge potential term, $E_{e}$, is given by Equation (8),

3. The distance potential, $E_{d 2}$ is a measure of the distance and shape of the mouth for a higher order boundary curve that can is defined similar to Equation (9):

$$
E_{d 2}=\left|h_{t b}-d\left(P_{i}, P_{j}\right)\right|
$$

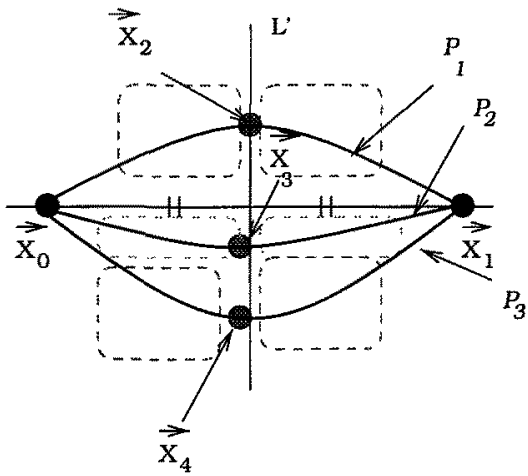

(a)

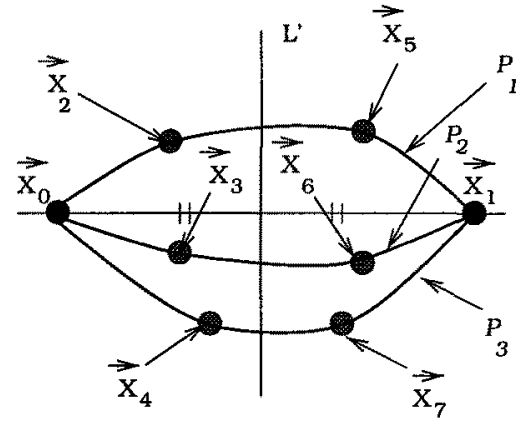

(b)

Fig. 2. A sketch of a deformable mouth template modeled with three boundary curves $P_{1}, P_{2}$ and $P_{3}$ for (a) initial model with 3 control points $X_{1}, \cdots, X_{3}$, and (b) final model with 4 control points $X_{1}, \cdots, X_{4}$.

For instance in the new model of the upper lip outline curve, the corner points $\mathbf{X}_{0}$ and $\mathbf{X}_{1}$ are retained, but $\mathbf{X}_{2}$ in the old model is replaced by two middle points $\mathbf{X}_{2}$ and $\mathbf{X}_{3}$ in the new model, as illustrated in Fig.2(b). However both new points $\mathbf{X}_{2}$ and $\mathbf{X}_{3}$ are initially located on $\mathbf{X}_{2}$ that of the old model. The dashed rectangular areas in Fig.2(a) shows an example of search area of control points of a new model. This methodology can be easily extended to further increase the number of control points. The secondary cost function is partially based on the potential energy functional employed in the old model. However in the secondary the corner potential terms are canceled since we assume that the corner points are optimally found in the primary optimization process. The secondary optimization process is mainly applied to locate the best control points for the new model. The new model captures more information about mouth outlines. If the cost function does not decrease in optimizing the new model, the old model is retained as the final model. It is always possible to increase the number of control points to achieve a control points leads to higher computational cost. However a higher order model is more prune to image noise and it is also computationally expensive. Therefore there is a trade-off between the order of the model, the amount of image noise and the speed of the algorithm. 


\section{Experiments}

We have tested our algorithm on a set of human face images obtained from Olivetti face image database, Cambridge. Our algorithm was implemented using $\mathrm{C}$ and run on SUN Sparc 2 workstation. To remove image background and also to locate an initial mouth region the boundary of a head is located using active contours. The mouth is modeled by three adaptive deformable templates. The model is initialized by parabolic curves using three control points and each control point is located using Equation (5). Then it is finely tuned by increasing the order of the model with adding more control points for each curve and optimizing Equation (10). We have found empirically that parabolic and cubic curve are usually sufficient to model various forms of a mouth image. Examples of mouth models superimposed on the mouth region are illustrated in Fig. 3.

\section{Conclusion}

We propose a new mouth model based on a hierarchical adaptive deformable template. This modeling scheme employed a priori knowledge of the shape of mouth contour to define a proper energy function. The energy function captures the properties of a mouth contour and its relation with other part of the mouth. Each contour line is modeled using a set of control points. The location of each control point is found by a greedy search algorithm. The model is tuned by increasing the number of control points to achieve a better model. The experimental showed satisfactory results on a database of human face images.

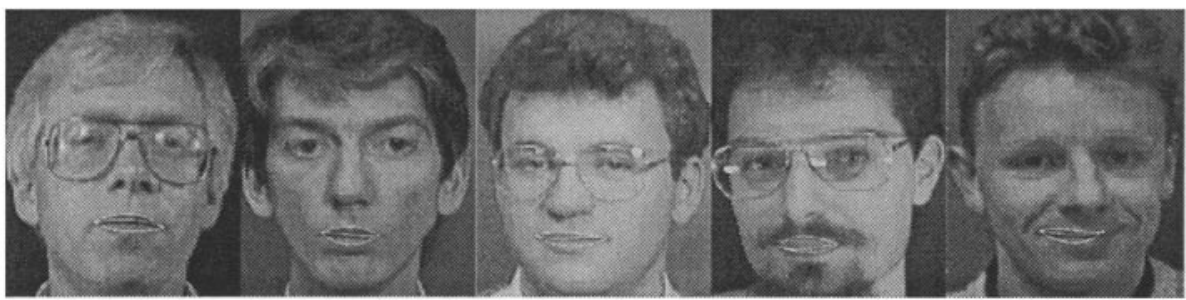

Fig. 3. The examples of mouth model using hierarchical adaptive deformable template.

\section{References}

1. A.Blake and A.L.Yuille, Deformable Templates in Active Vision, M.I.T. Press, Cambridge, (1992).

2. A.L.Yuille, P.W.Hallinan and D.S. Cohen, Feature Extraction from Faces using Deformable Templates, International Journal of Computer Vision, 8(2), 99-111, (1992). 
3. T. Kanade, Computer Recognition of Human Faces, Bikhauser Verlag, Basel and Stuttgart, (1977).

4. X.Xie, R.Sudhakar, and H.Zhuang, Corner Detection by a Cost Minimization Approach, Pattern Recognition, 26 (8): 1235-1243, (1993).

5. J.Serra, Image Analysis and Mathematical Morphology, Academic Press, New York, (1982).

6. M.Vezjak and M.Stefancic, An Anthropological Model for Automatic Recognition of the Male Face, Annals of Human Biology, 21 363, (1994).

7. A.L.Yuille, Deformable Templates for Face Recognition, Journal of Cognitive Neuroscience, 3 (1), 59-70, (1991).

8. M.Kass, A.Witkin and D.Terzopoulos, Snakes: Active Contour Models, in Proc. First Int. Conf. on Computer Vision, 259-269, London, (1995).

9. Kin-Man Lam, Computerized Human Face Recognition, $\mathrm{PhD}$ thesis, Department of Electrical Engineering, University of Sydney, Sydney, Australia, (1996).

10. A.R.Mirhosseini and H.Yan, An optimaly Fast Greedy Algorithm for Active Contours, IEEE International Symposium on Circuits and Systems, Hong Kong, (1997). 